\title{
Relativistic model for the nonmesonic weak decay of single-lambda hypernuclei
}

\author{
C E Fontoura ${ }^{1}$, F Krmpotić ${ }^{1,2}$, A P Galeão ${ }^{1}$, C De Conti ${ }^{3}$ and \\ G Krein ${ }^{1}$ \\ ${ }^{1}$ Instituto de Física Teórica, Universidade Estadual Paulista Rua Dr Bento Teobaldo \\ Ferraz, 271-Bloco II, 01140-070, São Paulo, SP, Brazil \\ ${ }^{2}$ Instituto de Física La Plata, Universidad Nacional de La Plata 1900 La Plata, \\ Argentina \\ ${ }^{3}$ Campus Experimental de Rosana, Universidade Estadual Paulista 19274-000 Rosana, \\ SP, Brazil \\ E-mail: gkrein@ift.unesp.br
}

Received 12 February 2016

Accepted for publication 17 February 2016

Published 22 March 2016

Communicated by Anthony W Thomas.

\begin{abstract}
Having in mind its future extension for theoretical investigations related to charmed nuclei, we develop a relativistic formalism for the nonmesonic weak decay (NMWD) of single- $\Lambda$ hypernuclei in the framework of the independentparticle shell model and with the dynamics represented by the $(\pi, K)$ onemeson-exchange model. Numerical results for the one-nucleon-induced transition rates of ${ }_{\Lambda}^{12} \mathrm{C}$ are presented and compared with those obtained in the analogous nonrelativistic calculation. There is satisfactory agreement between the two approaches, and the only noteworthy difference is that the ratio $\Gamma_{n} / \Gamma_{p}$ is appreciably higher and closer to the experimental value in the relativistic calculation. The ability of describing existing data, including the most recent ones, on NMWD of $\Lambda$-hypernuclei, warrants application of the formalism to evaluate similar decay processes in charmed nuclei.
\end{abstract}

Keywords: relativistic nuclear models, strange hypernuclei, charm hypernuclei, nonmesonic weak decay

\section{Introduction}

Investigations of exotic nuclear properties, such as large isospin (manifest in the so-called neutron-rich isotopes), or nontrivial values of flavor quantum numbers (strangeness, charm or beauty), are of continuous interest. The best-known nuclei within the last category are those 
where a $\Lambda$-hyperon, with strangeness $S=-1$, replaces one of the nucleons, giving to the composed system some quite unusual properties. Such nuclei are referred to as $\Lambda$-hypernuclei -for recent reviews, see [1,2].

One of the most remarkable properties of $\Lambda$-hypernuclei is the occurrence of the nonmesonic weak decay (NMWD), induced by the elementary process

$$
\Lambda+N \rightarrow n+N
$$

with $N=p$ (proton) or $n$ (neutron). This is the main decay channel for medium- and heavyweight hypernuclei-[3, 4] provide, respectively, reviews on recent theoretical and experimental developments in the study of hypernuclear decay. NMWD can only take place within the nuclear environment and is the unique opportunity offered by nature to access the strangeness-changing interaction between baryons. Its mean lifetime has been measured in several $\Lambda$-hypernuclei and found to be of the same order of magnitude as the full mean lifetime of $\Lambda$ in free space, $\tau_{\Lambda}=(2.632 \pm 0.020) \times 10^{-10} \mathrm{~s}[5]$.

The NMWD dynamics is frequently handled by nonrelativistic (NR) one-mesonexchange (OME) models. Such models are motivated by the fact that the $N N$ interaction at long distance is due to the one-pion-exchange, but with the difference that in NMWD the exchange processes occur with one strong and one weak vertex and can include other mesons in addition to the $\pi$, like the pseudoscalar $(K, \eta)$ and vector $\left(\rho, \omega, K^{*}\right)$ mesons [6-25]. The coupling constants at the strong vertices can be taken from different OME models for the $N N$ interaction, while those at the weak vertices can be extracted from free $\Lambda$ decay data and making use of soft meson theorems and $S U(6)_{\mathrm{W}}$ symmetry [7]. A recent study [23] indicates that $\pi$ and $K$ exchanges give the main contributions to the NMWD of $s$-shell hypernuclei.

Instead of implanting a $\Lambda$ in a nucleus one could also imagine to implant a charmed baryon, like e.g. a $\Lambda_{c}^{+}$, in view of the similarity between the quark structures of the strange and charmed hyperons, namely $\Lambda(u d s)$ and $\Lambda_{\mathrm{c}}^{+}(u d c)$. Such a possibility was in fact conjectured 40 years ago [26] and several authors in the succeeding decades have found, using different models for the interactions between nucleons and charmed hyperons, that such hypothetical exotic nuclei (including even bottom nuclei) could actually form a rich spectrum of bound states over a wide range of atomic numbers [27-38]. Like $\Lambda$-hypernuclei, $\Lambda_{\mathrm{c}}^{+}$-hypernuclei may also decay via a NMWD process. One example is [39]

$$
\Lambda_{\mathrm{c}}^{+}+n \rightarrow \Lambda+p
$$

which can be induced by the exchange of a $\pi, \rho$ or $K$ meson. Another possibility is

$$
\Lambda_{\mathrm{c}}^{+}+N \rightarrow p+N
$$

induced by the exchange of a $D$ meson. Experimentally, the literature only reports, inconclusively, the formation of three $\Lambda_{c}^{+}$-hypernuclei, observed in a series of emulsion experiments [40, 41]. But this situation can change in a few years, with the starting of operation of the FAIR facility in Germany and the Hadron Facility at JPARC in Japan.

There are, however, important differences between NMWD in $\Lambda$-hypernuclei and $\Lambda_{\mathrm{c}}^{+}$-hypernuclei. A first difference comes from the mean lifetimes of the two hyperons: $\tau_{\Lambda_{c}^{+}} \sim 10^{-3} \tau_{\Lambda}$. While the mean lifetime of the NMWD (1) is of the same order of magnitude of the full mean lifetime of $\Lambda$ in free space, no theoretical estimate has been made for the decays (2) and (3). In addition, while the free-space decay of $\Lambda$ is dominated by the pionic channels $\Lambda \rightarrow p \pi^{-}$and $\Lambda \rightarrow n \pi^{0}$, with other decay channels contributing a thousand times less, $\Lambda_{c}^{+}$decays in two semileptonic and numerous hadronic channels with $S=-1$ final states, having branching ratios of a few percent each. Also, decays into channels with $S=0$ and $S=+1$ are Cabibbo-suppressed by factors of the order of $10^{-1}-10^{-2}$ [5]. A second very 
important difference concerns the energy liberated in the decays, which is of the order of the mass difference $\Delta$ of the particles involved in the weak vertex: for the decay (2), $\Delta=M_{\Lambda_{\mathrm{c}}^{+}}-M_{\Lambda}=1170.9 \mathrm{MeV}$ and for the decay (3), $\Delta=M_{\Lambda_{\mathrm{c}}^{+}}-M_{\mathrm{N}}=1348.2 \mathrm{MeV}$, which should be compared to $\Delta=M_{\Lambda}-M_{\mathrm{N}}=177.3 \mathrm{MeV}$ for the decay (1). One consequence of such large energy releases is that NR approaches become inapplicable for the evaluation of NMWD transition matrix elements in charmed hypernuclei. In addition, a large energy release also implies that nuclear recoil cannot be neglected in the calculation of decay rates, particularly for light-weight nuclei. On the other hand, the interactions of the fast outgoing nucleons and/or hyperons with the residual nuclear system are expected to play a minor role.

In the present paper we develop a relativistic formalism for NMWD of hypernuclei within an independent-particle shell model (IPSM), and discuss the inclusion of recoil. Although the use of a relativistic model for the study of the structure of hypernuclei dates back to the late 1970s [42], so far little is known about the impact of a relativistic approach in the evaluation of NMWD rates. The first studies started 25 years ago [43] using single-particle bound-state wave functions obtained by solving the Dirac equation with static Lorentz-scalar and -vector Woods-Saxon potentials, and transition matrix elements calculated with a $(\pi, K)$ OME model. More recently, a similar approach was used in [44, 45], where the nuclear structure was described by a relativistic mesonic-mean-field model. Quark-meson coupling (QMC) models [46, 47], where mesonic fields couple directly to the light quarks composing the nucleons and hyperons, offer another interesting possibility-the model has been used to study strange [48-51] and charm [36-38] hypernuclei.

Our aim in the present paper is to set up a relativistic formalism for NMWD with the perspective of future applications to charmed hypernuclei. In other instances involving nuclear structure calculations at low and intermediate energies, it is often more convenient and simpler to use a relativistic approach than a NR one [52]; this seems to be also the case for NMWD-[53] presents a very complete review on relativistic approaches for the study of nuclear structure. Although our approach for the NMWD of hypernuclei shares similarities with previous publications, there are noteworthy differences:

(1) our final expressions for the decay rates do not involve angular momentum projection quantum numbers, since they have been summed over in closed form using the Racah algebra, which simplifies the numerical calculation;

(2) spectroscopic factors are evaluated in the second quantized formalism, as done for instance in [54], without recurring to the technique of coefficients of fractional parentage (c.f.p.), which is the standard antisymmetrization procedure in the first quantization framework, see e.g. [55];

(3) we discuss the inclusion of nuclear recoil, which is particularly important for NMWD of charmed hypernuclei.

The predictions of our formalism are compared with available data $[56,60]$ for the NMWD rates of the ${ }_{\Lambda}^{12} \mathrm{C}$ hypernucleus. In addition, we make a detailed comparison with results obtained in NR approaches that include the same ingredients (like short-range correlations (SRC) and OME model); such a comparison between the outcomes of analogous relativistic and NR approaches had not been done so far.

Our formalism is explained in section 2. We start in section 2.1 from the simplest scenario, corresponding to hypernuclei with closed-shell cores and ignoring recoil. This part is done in a strictly relativistic manner, while the next two steps are performed in analogy to NR calculations: first, in section 2.2, we generalize the formulation to hypernuclei with open- 
shell cores; and secondly, in section 2.3, the recoil effect is discussed. Subsequently, in section 3, our numerical results for the decay rates of ${ }_{\Lambda}^{12} \mathrm{C}$ are presented and compared to those of NR calculations using a similar model [61, 62]. They are also compared with those of previous relativistic calculations and confronted with the experimental data, and a few conclusions are drawn. Finally, in section 4, a general summary is given. The appendices collect details of some calculations.

\section{Relativistic decay rate}

To derive the NMWD rate we start from the Fermi Golden Rule. For a hypernucleus in its ground state with spin $J_{\mathrm{I}}$ and total rest energy $E_{\mathrm{I}}$ decaying into (i) two free nucleons, with asymptotic kinetic energies $\left(T_{1}, T_{2}\right)$, spin projections $\left(s_{1}, s_{2}\right)$, and isospin projections $\left(t_{1}, t_{2}\right)$ and (ii) the residual $(A-2)$-system, with spin $J_{\mathrm{F}}$, total rest energy $E_{\mathrm{F}}$, and kinetic energy of recoil $T_{\mathrm{R}}$, reads

$$
\Gamma_{n m}=\frac{2 \pi}{\left(2 J_{\mathrm{I}}+1\right)} \sum_{M_{\mathrm{I}} J_{\mathrm{F}} M_{\mathrm{F}}} \sum_{s_{1} s_{2} t_{1} t_{2}} \int \frac{\mathrm{d} \mathbf{p}_{1}}{(2 \pi)^{3}} \frac{\mathrm{d} \mathbf{p}_{2}}{(2 \pi)^{3}} \delta\left(E_{\mathrm{I}}-E_{\mathrm{F}}-\mathcal{E}\right)\left|\overline{\mathcal{M}}_{\mathrm{FI}}\right|^{2},
$$

where we are using a compact notation for the transition amplitude whose explicit momentum and spin and angular momentum dependence is

$$
\overline{\mathcal{M}}_{\mathrm{FI}} \equiv \overline{\mathcal{M}}\left(\mathbf{p}_{1} \mathbf{p}_{2} s_{1} s_{2} t_{1} t_{2} J_{\mathrm{F}} M_{\mathrm{F}}, J_{\mathrm{I}} M_{\mathrm{I}}\right)
$$

with $\overline{\mathcal{M}}=\left(1-P_{12}\right) \mathcal{M} / \sqrt{2}$ being the antisymmetrized and normalized relativistic matrix element that is specified below. In addition, $\mathcal{E}=2 M_{\mathrm{N}}-T_{\mathrm{R}}-T_{2}-T_{1}, M_{\mathrm{N}}$ is the nucleon mass, $p_{i}=\sqrt{E_{i}^{2}-M_{\mathrm{N}}^{2}}$ and $E_{i}=T_{i}+M_{\mathrm{N}}$ are the asymptotic momenta and total energies of the outgoing particles $(i=1,2)$. We use unitary, as opposed to covariant, normalization for the momentum eigenspinors; for details see section 2.2 of [63]. We average over the spin projections $M_{\mathrm{I}}$ of the initial hypernucleus and sum over the final spin projections $M_{\mathrm{F}}$.

For the nuclear structure, the IPSM is used, while the dynamics is described by an OME potential containing always one weak vertex $\mathrm{W}$ and and one strong vertex $\mathrm{S}$, as illustrated in figure 1. In the IPSM it is assumed that: (i) the initial hypernuclear state can be approximated as a $\Lambda$-hyperon in the single-particle state $j_{\Lambda}=1 s_{1 / 2}$ weakly coupled to an $(A-1)$ nuclear core of spin $J_{\mathrm{C}}$ and total rest energy $E_{\mathrm{C}}$, i.e., $\left|J_{\mathrm{I}}\right\rangle \equiv\left|\left(J_{\mathrm{C}} j_{\Lambda}\right) J_{\mathrm{I}}\right\rangle$, having energy $E_{\mathrm{I}}=E_{\mathrm{C}}+\varepsilon_{j_{\Lambda}}+M_{\Lambda}$; (ii) the nucleon $N$ inducing the decay is in the single-particle state $j_{N}$ $(j \equiv n l j)$; (iii) the final residual nuclear states have the form $\left|J_{\mathrm{F}}\right\rangle \equiv\left|\left(J_{\mathrm{C}} j_{\mathrm{N}}^{-1}\right) J_{\mathrm{F}}\right\rangle$ with energy $E_{\mathrm{F}}=E_{\mathrm{C}}-\varepsilon_{j_{\mathrm{N}}}-M_{\mathrm{N}}$; (iv) the liberated kinetic energy is

$$
T_{\mathrm{R}}+T_{2}+T_{1}=E_{\mathrm{I}}-E_{\mathrm{F}}-2 M_{\mathrm{N}} \equiv \Delta_{j_{\mathrm{N}}}=\Delta+\varepsilon_{j_{\Lambda}}+\varepsilon_{j_{\mathrm{N}}},
$$

where $\Delta=M_{\Lambda}-M_{\mathrm{N}}=177 \mathrm{MeV}$, and the $\varepsilon$ s are single-particle energies.

\subsection{Hypernuclei with doubly closed shell cores and without recoil}

Taking the simplest possible case in equation (4), we will start with hypernuclei whose cores contain only doubly closed subshells, as, for instance, ${ }_{\Lambda}^{5} \mathrm{He},{ }_{\Lambda}^{13} \mathrm{C},{ }_{\Lambda}^{17} \mathrm{O}$, and we will omit the recoil effect. Thus, $J_{\mathrm{C}}=0, J_{\mathrm{I}}=j_{\Lambda}, M_{\mathrm{I}}=m_{\Lambda}, J_{\mathrm{F}}=j_{\mathrm{N}}, M_{\mathrm{F}}=m_{\mathrm{N}}$, and the transition amplitudeis, up to a global sign, just the two-body T-matrix for the direct OME process. When a pseudoscalar coupling is considered for the strong vertex, one has for the pion plus kaon meson exchange, $\mathcal{M}_{\mathrm{FI}}=\mathcal{M}_{\mathrm{FI}}^{\pi}+\mathcal{M}_{\mathrm{FI}}^{K}$, with 


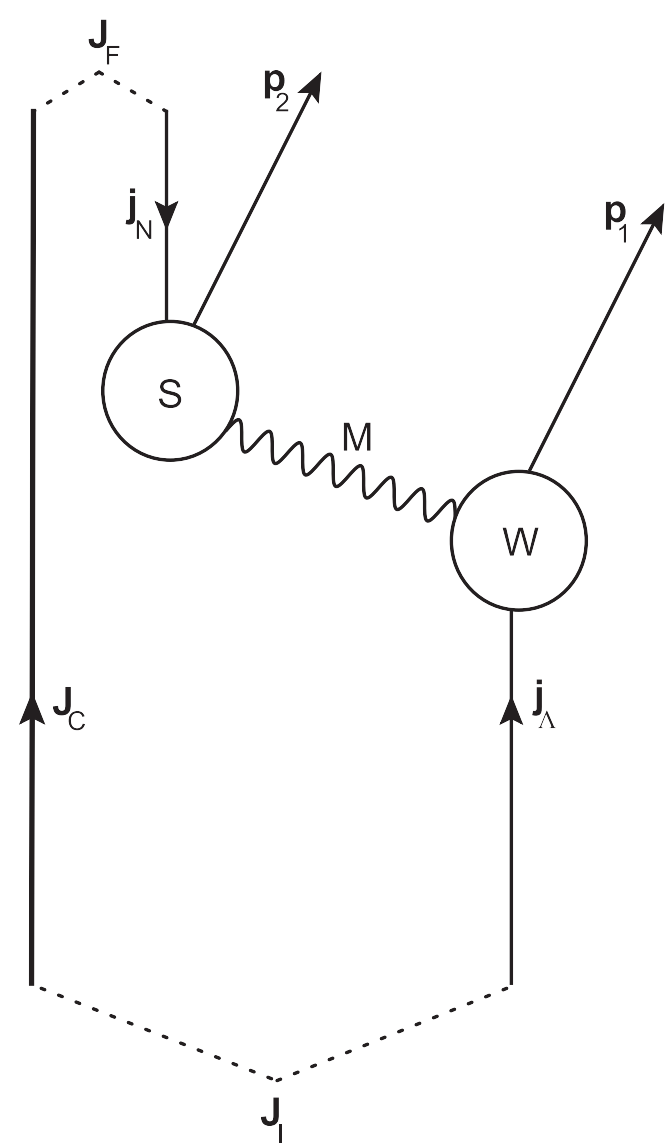

Figure 1. Diagrammatic representation of the hypernuclear NMWD from the initial state $\left|J_{\mathrm{I}}\right\rangle \equiv\left|\left(J_{\mathrm{C}} j_{\Lambda}\right) J_{\mathrm{I}}\right\rangle$ to the final state $\left|J_{\mathrm{F}}\right\rangle \equiv\left|\left(J_{\mathrm{C}} j_{\mathrm{N}}^{-1}\right) J_{\mathrm{F}}\right\rangle$, while two nucleons with momenta $\mathbf{p}_{1}$ and $\mathbf{p}_{2}$ are emitted into the continuum. $\mathrm{S}$ and $\mathrm{W}$ are the strong and the weak vertices, respectively, and $\mathrm{M}$ is a nonstrange meson. For a strange meson, the natures of the two vertices should be interchanged.

$\mathcal{M}_{\mathrm{FI}}^{\pi}=\int \mathrm{d} \mathbf{x} \mathrm{d} \mathbf{y} \bar{\psi}_{\mathbf{p}_{1} s_{1}}(\mathbf{x}) \Gamma_{\mathrm{W}}^{\pi}\left(t_{1}, t_{2}\right) \Psi_{j_{\Lambda} m_{\Lambda}}(\mathbf{x}) \Delta^{\pi}(|\mathbf{x}-\mathbf{y}|) \bar{\psi}_{\mathbf{p}_{2} s_{2}}(\mathbf{y}) \gamma_{5} \Psi_{j_{\mathrm{N}} m_{\mathrm{N}}}(\mathbf{y})$,

$\mathcal{M}_{\mathrm{FI}}^{K}=\int \mathrm{d} \mathbf{x d} \mathbf{y} \bar{\psi}_{\mathbf{p}_{1} s_{1}}(\mathbf{x}) \gamma_{5} \Psi_{j_{\Lambda} m_{\Lambda}}(\mathbf{x}) \Delta^{K}(|\mathbf{x}-\mathbf{y}|) \bar{\psi}_{\mathbf{p}_{2} s_{2}}(\mathbf{y}) \Gamma_{\mathrm{W}}^{K}\left(t_{1}, t_{2}\right) \Psi_{j_{\mathrm{N}} m_{\mathrm{N}}}(\mathbf{y})$,

where we have defined $\Gamma_{\mathrm{W}}^{\mathrm{M}}\left(t_{1}, t_{2}\right), M=(\pi, K)$, as being

$$
\Gamma_{\mathrm{W}}^{\mathrm{M}}\left(t_{1}, t_{2}\right)=\mathcal{A}^{\mathrm{M}}\left(t_{1}, t_{2}\right)-\mathcal{B}^{\mathrm{M}}\left(t_{1}, t_{2}\right) \gamma_{5},
$$

with

$$
\begin{aligned}
& \mathcal{A}^{\pi}\left(t_{1}, t_{2}\right)=G_{\mathrm{F}} m_{\pi}^{2} g_{\pi N N} A I\left(t_{1}, t_{2}\right), \\
& \mathcal{A}^{K}\left(t_{1}, t_{2}\right)=G_{\mathrm{F}} m_{\pi}^{2} g_{K \Lambda N}\left[A_{1} I\left(t_{1}, t_{2}\right)+A_{0} K\left(t_{1}, t_{2}\right)\right], \\
& \mathcal{B}^{\pi}\left(t_{1}, t_{2}\right)=G_{\mathrm{F}} m_{\pi}^{2} g_{\pi N N} B I\left(t_{1}, t_{2}\right), \\
& \mathcal{B}^{K}\left(t_{1}, t_{2}\right)=G_{\mathrm{F}} m_{\pi}^{2} g_{K \Lambda N}\left[B_{1} I\left(t_{1}, t_{2}\right)+B_{0} K\left(t_{1}, t_{2}\right)\right],
\end{aligned}
$$


where $I\left(t_{1}, t_{2}\right)$ and $K\left(t_{1}, t_{2}\right)$ are, respectively, the isovector and isoscalar isospin factors:

$$
\begin{aligned}
I\left(t_{1}, t_{2}\right) & =\left\langle t_{1}\left|\tau_{1}\right| t_{\Lambda}=-1 / 2\right\rangle\left\langle t_{2}\left|\tau_{2}\right| t_{\mathrm{N}}\right\rangle, \\
K\left(t_{1}, t_{2}\right) & =\left\langle t_{1}\left|1_{1}\right| t_{\Lambda}=-1 / 2\right\rangle\left\langle t_{2}\left|1_{2}\right| t_{\mathrm{N}}\right\rangle .
\end{aligned}
$$

Here, $G_{\mathrm{F}} m_{\pi}^{2}=2.21 \times 10^{-7}$, with $G_{\mathrm{F}}$ being the Fermi weak constant and $m_{\pi}$ the pion mass, while $g_{\pi N N}=13.3$ and $g_{K \Lambda N}=-14.1$ are the strong vertex couplings [64]. The pion parityviolating (PV) and parity-conserving (PC) weak coupling constants are adjusted to the free $\Lambda$ decay giving, respectively, $A=1.05$ and $B=-7.15$, while the kaon weak couplings are

$$
A_{0}=\frac{C_{K}^{\mathrm{PV}}}{2}+D_{K}^{\mathrm{PV}}, A_{1}=\frac{C_{K}^{\mathrm{PV}}}{2}, B_{0}=\frac{C_{K}^{\mathrm{PC}}}{2}+D_{K}^{\mathrm{PC}}, B_{1}=\frac{C_{K}^{\mathrm{PC}}}{2},
$$

with $C_{K}^{\mathrm{PV}}=0.76, C_{K}^{\mathrm{PC}}=-18.9, D_{K}^{\mathrm{PV}}=2.09$, and $D_{K}^{\mathrm{PC}}=6.63$, have been estimated theoretically in [7]. The propagator, $\Delta^{\mathrm{M}}(r)(M=\pi, K ; r=|\mathbf{x}-\mathbf{y}|)$, depends on the energy $q^{0}$ carried by the exchanged meson, whose value is fixed by energy conservation (see discussion in section 3), and incorporates dipole form factors, with a cutoff parameter $\Lambda_{\mathrm{M}}$, that are attached to each meson-baryon vertex. Defining the quantities

$$
\lambda_{\mathrm{M}}\left(q^{0}\right)=\sqrt{\left|\left(q^{0}\right)^{2}-m_{\mathrm{M}}^{2}\right|}, \Lambda_{\mathrm{M}}\left(q^{0}\right)=\sqrt{\Lambda_{\mathrm{M}}^{2}-\left(q^{0}\right)^{2}},
$$

one can write $\Delta^{\mathrm{M}}(r)$ as $[44,45]$ :

(i) For $\left(q^{0}\right)^{2}<m_{\mathrm{M}}^{2}$,

$$
\Delta^{\mathrm{M}}(r)=-\frac{\mathrm{e}^{-\lambda_{\mathrm{M}}\left(q^{0}\right) r}}{4 \pi r}+\left[\frac{1}{4 \pi r}+\frac{\Lambda_{\mathrm{M}}^{2}-m_{\mathrm{M}}^{2}}{8 \pi \Lambda_{\mathrm{M}}\left(q^{0}\right)}\right] \mathrm{e}^{-\Lambda_{\mathrm{M}}\left(q^{0}\right) r},
$$

(ii) For $m_{\mathrm{M}}^{2}<\left(q^{0}\right)^{2}<\Lambda_{\mathrm{M}}^{2}$,

$$
\Delta^{\mathrm{M}}(r)=-\frac{\mathrm{e}^{\mathrm{i} \lambda} \lambda_{\mathrm{M}}\left(q^{0}\right) r}{4 \pi r}
$$

Note that for the kinematical situation (ii), the propagator is complex and can have an oscillatory behavior.

The state of each ejected nucleon, with asymptotic momentum $\mathbf{p}$ and spin projection $s$, will be approximated by a Dirac plane wave, which is expanded in spherical partial waves as follows ([65] appendix D):

$$
\psi_{\mathbf{p} s}(\mathbf{r})=\sum_{\kappa m}\langle\hat{\mathbf{p}} s \mid \kappa m\rangle^{*} \psi_{p \kappa m}(\mathbf{r})
$$

with

$$
\langle\hat{\mathbf{p}} s \mid \kappa m\rangle^{*}=4 \pi \mathrm{i}^{l} \sum_{\mu}\left(l \mu \frac{1}{2} s \mid j m\right) Y_{l \mu}^{*}(\hat{\mathbf{p}})
$$

and

$$
\psi_{p \kappa m}(\mathbf{r})=\left(\begin{array}{c}
f_{p \kappa}(r) \Phi_{\kappa m}(\hat{\mathbf{r}}) \\
-\mathrm{i} g_{p \kappa}(r) \Phi_{-\kappa m}(\hat{\mathbf{r}})
\end{array}\right) \equiv\left(\begin{array}{c}
{ }^{\uparrow} \psi_{p \kappa m}(\mathbf{r}) \\
-\mathrm{i} \downarrow \psi_{p \kappa m}(\mathbf{r})
\end{array}\right),
$$


where the radial partial waves are, in unitary normalization

$$
\begin{aligned}
& f_{p \kappa}(r)=\sqrt{\frac{E+M_{\mathrm{N}}}{2 E}} j_{l_{\kappa}}(p r), \\
& g_{p \kappa}(r)=-\operatorname{sgn}(\kappa) \sqrt{\frac{E-M_{\mathrm{N}}}{2 E}} j_{\bar{l}_{\kappa}}(p r),
\end{aligned}
$$

with $\kappa= \pm 1, \pm 2, \ldots, j_{\kappa}=|\kappa|-1 / 2$,

$$
l_{\kappa}= \begin{cases}\kappa & \text { for } \kappa>0 \\ -\kappa-1 & \text { for } \kappa<0\end{cases}
$$

and $\bar{l}_{\kappa}=l_{-\kappa}$. To change to covariant normalization, used e.g. in [43-45], make the replacement $\sqrt{2 E} \rightarrow \sqrt{2 M_{\mathrm{N}}}$ in equations (19) and (20) and insert the factor $M_{\mathrm{N}}^{2} /\left(E_{1} E_{2}\right)$ in equation (4). The angular part is written, in standard notation, as

$$
\Phi_{\kappa m}(\hat{\mathbf{r}})=\sum_{s \mu}\left(l \mu \frac{1}{2} s \mid j m\right) Y_{l \mu}(\hat{\mathbf{r}}) \chi_{\mathrm{s}}
$$

and the expansion coefficients $\langle\hat{\mathbf{p}} s \mid \kappa m\rangle^{*}$ fulfill the following relations

$$
\begin{aligned}
& \sum_{\mathrm{s}} \int \mathrm{d} \hat{\mathbf{p}}\langle\hat{\mathbf{p}} s \mid \kappa m\rangle^{*}\left\langle\hat{\mathbf{p}} s \mid \kappa^{\prime} m^{\prime}\right\rangle=(4 \pi)^{2} \delta_{\kappa \kappa^{\prime}} \delta_{m m^{\prime}}, \\
& 2 \hat{j}^{2} \delta_{j j^{\prime}} \sum_{s m} \int \mathrm{d} \hat{\mathbf{p}}\langle\hat{\mathbf{p}} s \mid \kappa m\rangle^{*}\left\langle\hat{\mathbf{p}} s \mid \kappa^{\prime} m\right\rangle \ldots=(4 \pi)^{2} \delta_{\kappa \kappa^{\prime}} \int_{-1}^{1} \mathrm{~d} \cos \theta \ldots,
\end{aligned}
$$

where we are using the notation $\hat{j}=\sqrt{2 j+1}$. The first of these relations can be easily verified, while the second one is shown in appendix A. The bound-state, single-particle, wave functions read

$$
\Psi_{\kappa m}(\mathbf{r})=\frac{1}{r}\left(\begin{array}{c}
F_{\kappa}(r) \Phi_{\kappa m}(\hat{\mathbf{r}}) \\
-\mathrm{i} G_{\kappa}(r) \Phi_{-\kappa m}(\hat{\mathbf{r}})
\end{array}\right) \equiv\left(\begin{array}{c}
\uparrow \Psi_{\kappa m}(\mathbf{r}) \\
-\mathrm{i} \downarrow \Psi_{\kappa m}(\mathbf{r})
\end{array}\right) .
$$

As explained in appendix B, they are evaluated as in ([66], equation (16)).

To simplify the presentation of formulas in the analytical development of the expression of the decay rate in equation (4), the intermediate steps will be exhibited only for $\mathcal{M}_{\mathrm{FI}}^{\pi}$ :

$$
\mathcal{M}_{\mathrm{FI}}^{\pi}=\sum_{\substack{\kappa_{1} m_{1} \\ \kappa_{2} m_{2}}}\left\langle\hat{\mathbf{p}}_{1} s_{1} \mid \kappa_{1} m_{1}\right\rangle\left\langle\hat{\mathbf{p}}_{2} s_{2} \mid \kappa_{2} m_{2}\right\rangle\left\langle F\left|\Delta^{\pi}\right| I\right\rangle,
$$

where we are using the compact notation:

$$
\begin{aligned}
\left\langle F\left|\Delta^{\pi}\right| I\right\rangle \equiv & \int \mathrm{d} \mathbf{x d} \mathbf{y} \bar{\psi}_{p_{1} \kappa_{1} m_{1}}(\mathbf{x}) \Gamma_{\mathrm{W}}^{\pi}\left(t_{1}, t_{2}\right) \Psi_{j_{\Lambda} m_{\Lambda}}(\mathbf{x}) \\
& \times \Delta^{\pi}(|\mathbf{x}-\mathbf{y}|) \bar{\psi}_{p_{2} \kappa_{2} m_{2}}(\mathbf{y}) \gamma_{5} \Psi_{j_{\mathrm{N}} m_{\mathrm{N}}}(\mathbf{y}) .
\end{aligned}
$$

Introducing these expansions in the expression for the decay rate in equation (4) gives rise to auxiliary quantities such as

$$
S^{\pi}\left(p_{1} t_{1}, p_{2} t_{2}\right) \equiv \sum_{\substack{m_{\Lambda} m_{\mathrm{N}} \\ s_{1} s_{2}}} \int \mathrm{d} \hat{\mathbf{p}}_{1} \mathrm{~d} \hat{\mathbf{p}}_{2} \delta\left(\Delta_{j_{\mathrm{N}}}-T_{1}-T_{2}-T_{\mathrm{R}}\right)\left|\mathcal{M}_{\mathrm{FI}}^{\pi}\right|^{2},
$$

in which we evaluate all the summations over angular momentum projection quantum numbers and angular integrations. Neglecting recoil, i.e., setting $T_{\mathrm{R}}=0$, we can use 
equation (23) for both outgoing particles getting

$$
S^{\pi}\left(p_{1} t_{1}, p_{2} t_{2}\right)=(4 \pi)^{4} \sum_{\substack{m_{\Lambda} m_{\mathrm{N}} \\ \kappa_{1} m_{1} \kappa_{2} m_{2}}} \delta\left(\Delta_{j_{\mathrm{N}}}-T_{1}-T_{2}\right)\left|\left\langle F\left|\Delta^{\pi}\right| I\right\rangle\right|^{2} .
$$

Now we perform the angular momentum couplings $\vec{J}=\vec{j}_{\Lambda}+\vec{j}_{\mathrm{N}}$ and $\vec{J}^{\prime}=\vec{j}_{1}+\vec{j}_{2}$. As $\Delta^{\pi}$ is rotationally invariant, it turns out that $J=J^{\prime}$, which leads to

$$
\left\langle F\left|\Delta^{\pi}\right| I\right\rangle=\sum_{J M}\left(j_{1} m_{1} j_{2} m_{2} \mid J M\right)\left(j_{\Lambda} m_{\Lambda} j_{\mathrm{N}} m_{\mathrm{N}} \mid J M\right)\left\langle F\left|\Delta^{\pi}\right| I\right\rangle_{J}
$$

and

$$
S^{\pi}\left(p_{1} t_{1}, p_{2} t_{2}\right)=(4 \pi)^{4} \sum_{\kappa_{1} \kappa_{2} J} \hat{J}^{2} \delta\left(\Delta_{j_{\mathrm{N}}}-T_{1}-T_{2}\right)\left|\left\langle F\left|\Delta^{\pi}\right| I\right\rangle_{J}\right|^{2}
$$

where the coupled matrix element of the pion propagator is explicitly given by

$$
\begin{aligned}
\left\langle F\left|\Delta^{\pi}\right| I\right\rangle_{J}= & -\mathrm{i} \int \mathrm{d} \mathbf{x} \mathrm{d} \mathbf{y}\left\{\left[\mathcal{A}^{\pi}\left(t_{1}, t_{2}\right) \rho_{A}(\mathbf{x})+\mathcal{B}^{\pi}\left(t_{1}, t_{2}\right) \rho_{B}(\mathbf{x})\right]\right. \\
& \left.\times \Delta^{\pi}(|\mathbf{x}-\mathbf{y}|) \rho_{\mathrm{C}}(\mathbf{y})\right\}_{\left(j_{1} j_{2}, j_{\Lambda} j_{\mathrm{N}} ; J\right)},
\end{aligned}
$$

where $\left(j_{1} j_{2}, j_{\Lambda} j_{\mathrm{N}} ; J\right)$ indicates the angular momentum couplings and the densities $\rho_{A}, \rho_{B}$ and $\rho_{\mathrm{C}}$ are given by

$$
\begin{aligned}
& \rho_{A}(\mathbf{x})={ }^{\uparrow} \psi_{p_{1} \kappa_{1}}^{*}(\mathbf{x})^{\uparrow} \Psi_{\kappa_{\Lambda}}(\mathbf{x})-\downarrow{ }^{\prime} \psi_{p_{1} \kappa_{1}}^{*}(\mathbf{x}){ }^{\downarrow} \Psi_{\kappa_{\Lambda}}^{*}(\mathbf{x}), \\
& \rho_{B}(\mathbf{x})={ }^{\top} \psi_{p_{1} \kappa_{1}}^{*}(\mathbf{x})^{\downarrow} \Psi_{\kappa_{\Lambda}}(\mathbf{x})+{ }^{\downarrow} \psi_{p_{1} \kappa_{1}}^{*}(\mathbf{x})^{\uparrow} \Psi_{\kappa_{\Lambda}}^{*}(\mathbf{x}), \\
& \rho_{\mathrm{C}}(\mathbf{x})={ }^{\top} \psi_{p_{2} \kappa_{2}}^{*}(\mathbf{x})^{\downarrow} \Psi_{\kappa_{\mathrm{N}}}(\mathbf{x})+\downarrow \downarrow \psi_{p_{2} \kappa_{2}}^{*}(\mathbf{x})^{\uparrow} \Psi_{\kappa_{\mathrm{N}}}^{*}(\mathbf{x}) .
\end{aligned}
$$

At this point it is convenient to perform the tensor expansion of the propagators $\Delta^{\pi}(|\mathbf{x}-\mathbf{y}|)$ in the way done by de-Shalit and Talmi ([55], section 21) for two-body interactions, i.e.,

$$
\Delta^{\pi}(|\mathbf{x}-\mathbf{y}|)=\sum_{L} \Delta_{L}^{\pi}(x, y)\left[Y_{L}(\hat{\mathbf{x}})_{L} \cdot Y(\hat{\mathbf{y}})_{L}\right]
$$

where

$$
\Delta_{L}^{\pi}(x, y)=2 \pi \int \Delta^{\pi}(|\mathbf{x}-\mathbf{y}|) P_{L}\left(\cos \theta_{x y}\right) \mathrm{d}\left(\cos \theta_{x y}\right)
$$

and

$\left\langle\kappa_{1} \kappa_{2} J\left|\left[Y_{L}(\hat{\mathbf{x}}) Y_{L}(\hat{\mathbf{y}})\right]\right| \kappa_{\Lambda} \kappa_{\mathrm{N}} J\right\rangle=(-)^{j_{2}+j_{\Lambda}+J}\left\{\begin{array}{lll}j_{1} & j_{2} & J \\ j_{\mathrm{N}} & j_{\Lambda} & L\end{array}\right\}\left\langle\kappa_{1}\left\|Y_{L}\right\| \kappa_{\Lambda}\right\rangle\left\langle\kappa_{2}\left\|Y_{L}\right\| \kappa_{\mathrm{N}}\right\rangle$

The reduced matrix elements

$$
\left\langle\kappa\left\|Y_{L}\right\| \kappa^{\prime}\right\rangle=(4 \pi)^{-1 / 2}(-)^{j-1 / 2} \hat{j} \hat{j}^{\prime} \hat{L}\left(\begin{array}{ccc}
j & L & j^{\prime} \\
-\frac{1}{2} & 0 & \frac{1}{2}
\end{array}\right) \frac{1+(-)^{l+l^{\prime}+L}}{2}
$$

and

$$
\left\langle-\kappa\left\|Y_{L}\right\| \kappa^{\prime}\right\rangle=(4 \pi)^{-1 / 2}(-)^{j-1 / 2} \hat{j} \hat{j}^{\prime} \hat{L}\left(\begin{array}{ccc}
j & L & j^{\prime} \\
-\frac{1}{2} & 0 & \frac{1}{2}
\end{array}\right) \frac{1+(-)^{\bar{l}+l^{\prime}+L}}{2},
$$


Table 1. Isospin factors the for direct (D) and exchange (E) terms of the matrix element in equation (45).

\begin{tabular}{ccccc}
\hline & \multicolumn{2}{c}{$I$} & \multicolumn{2}{c}{$K$} \\
\hline & $n$ & $p$ & $n$ & $p$ \\
\hline $\mathrm{D}$ & 1 & -1 & 1 & 1 \\
$\mathrm{E}$ & 1 & 2 & 1 & 0 \\
\hline
\end{tabular}

fulfill the symmetry relations $\left\langle\kappa\left\|Y_{L}\right\| \kappa^{\prime}\right\rangle=\left\langle\kappa^{\prime}\left\|Y_{L}\right\| \kappa\right\rangle$, and $\left\langle\kappa\left\|Y_{L}\right\|-\kappa^{\prime}\right\rangle=\left\langle-\kappa\left\|Y_{L}\right\| \kappa^{\prime}\right\rangle$. It is then easy to demonstrate that

$$
\left\langle F\left|\Delta^{\pi}\right| I\right\rangle_{J}=\sum_{L}(-)^{j_{2}+j_{\Lambda}+J}\left\{\begin{array}{lll}
j_{1} & j_{2} & J \\
j_{\mathrm{N}} & j_{\Lambda} & L
\end{array}\right\}\left\langle F\left|\Delta_{L}^{\pi}\right| I\right\rangle,
$$

where

$$
\begin{aligned}
\left\langle F\left|\Delta_{L}^{\pi}\right| I\right\rangle= & \int \mathrm{d} x \mathrm{~d} y x y\left[\mathcal{B}^{\pi}\left(t_{1}, t_{2}\right) B_{\kappa_{1} \kappa_{\Lambda}}^{L}\left(x p_{1}\right)\right. \\
& \left.-\mathrm{i} \mathcal{A}^{\pi}\left(t_{1}, t_{2}\right) A_{\kappa_{1} \kappa_{\Lambda}}^{L}\left(x p_{1}\right)\right] \Delta_{L}^{\pi}(x, y) C_{\kappa_{2} \kappa_{\mathrm{N}}}^{L}\left(y p_{2}\right),
\end{aligned}
$$

with

$$
\begin{aligned}
A_{\kappa \kappa_{\Lambda}}^{L}(r p) & =\left[f_{p \kappa}(r) F_{\kappa_{\Lambda}}(r)-g_{p \kappa}(r) G_{\kappa_{\Lambda}}(r)\right]\left\langle\kappa\left\|Y_{L}\right\| \kappa_{\Lambda}\right\rangle, \\
B_{\kappa \kappa_{\Lambda}}^{L}(r p) & =\left[f_{p \kappa}(r) G_{\kappa_{\Lambda}}(r)+g_{p \kappa}(r) F_{\kappa_{\Lambda}}(r)\right]\left\langle-\kappa\left\|Y_{L}\right\| \kappa_{\Lambda}\right\rangle, \\
C_{\kappa \kappa_{\mathrm{N}}}^{L}(r p) & =\left[f_{p \kappa}(r) G_{\kappa_{\mathrm{N}}}(r)+g_{p \kappa}(r) F_{\kappa_{\mathrm{N}}}(r)\right]\left\langle-\kappa\left\|Y_{L}\right\| \kappa_{\mathrm{N}}\right\rangle .
\end{aligned}
$$

The $K$ meson is incorporated through the substitution $\Delta_{L}^{\pi} \rightarrow \Delta_{L}=\Delta_{L}^{\pi}+\Delta_{L}^{K}$ in equation (40), with

$$
\begin{aligned}
\left\langle F\left|\Delta_{L}^{K}\right| I\right\rangle= & \int \mathrm{d} x \mathrm{~d} y x y B_{\kappa_{1} \kappa_{\Lambda}}^{L}\left(x p_{1}\right) \Delta_{L}^{K}(x, y)\left[\mathcal{B}^{K}\left(t_{1}, t_{2}\right) C_{\kappa_{2} \kappa_{\mathrm{N}}}^{L}\left(y p_{2}\right)\right. \\
& \left.-\mathrm{i} \mathcal{A}^{K}\left(t_{1}, t_{2}\right) D_{\kappa_{2} \kappa_{\mathrm{N}}}^{L}\left(y p_{2}\right)\right]
\end{aligned}
$$

where

$$
D_{\kappa \kappa_{\mathrm{N}}}^{L}(r p)=\left[f_{p \kappa}(r) F_{\kappa_{\mathrm{N}}}(r)-g_{p \kappa}(r) G_{\kappa_{\mathrm{N}}}(r)\right]\left\langle\kappa\left\|Y_{L}\right\| \kappa_{\mathrm{N}}\right\rangle .
$$

Clearly the above substitution must be accompanied by the replacement $\Delta^{\pi} \rightarrow \Delta=\Delta^{\pi}+\Delta^{K}$ in equation (39), giving

$$
\langle F|\Delta| I\rangle_{J}=\sum_{L}(-)^{j_{2}+j_{\Lambda}+J}\left\{\begin{array}{lll}
j_{1} & j_{2} & J \\
j_{\mathrm{N}} & j_{\Lambda} & L
\end{array}\right\}\left\langle F\left|\Delta_{L}\right| I\right\rangle .
$$

Finally, the expression for the decay rate in equation (4) can be written as

$$
\Gamma_{\mathrm{N}}=\frac{8}{\pi} \sum_{\substack{j_{\mathrm{N}} t_{1} t_{2} \\ \kappa_{1} \kappa_{2} J}} \frac{\hat{J}^{2}}{\hat{j}_{\Lambda}^{2}} \int \mathrm{d} p_{1} \mathrm{~d} p_{2} p_{1}^{2} p_{2}^{2} \delta\left(\Delta_{j_{\mathrm{N}}}-T_{1}-T_{2}\right)\left|\langle\bar{F}|\Delta| I\rangle_{J}\right|^{2},
$$


where

$$
\langle\bar{F}|\Delta| I\rangle_{J}=\frac{1}{\sqrt{2}}\left(\langle F|\Delta| I\rangle_{J}-(-)^{j_{1}+j_{2}-J}\left[\left(p_{1} \kappa_{1} t_{1}\right) \leftrightarrow\left(p_{2} \kappa_{2} t_{2}\right)\right]\right)
$$

stands for the antisymmetrized and normalized matrix element, with the isospins included. The isospin factors for the direct and exchange terms of the matrix element in equation (45) are listed in table 1.

It is worth noting that the matrix elements $\langle\bar{F}|\Delta| I\rangle$ are in general complex, as seen from equations (40) and (42). However, in the usual kinematical regime $\left(q^{0}\right)^{2}<m_{\mathrm{M}}^{2}$, see equation (14), they are always either real or purely imaginary because there is no set of quantum numbers for which PC and PV contributions interfere with each other.

To exploit the implications of the delta function in equation (45) we make use of the relation

$$
p_{i}^{2} \mathrm{~d} p_{i}=E_{i} \sqrt{E_{i}^{2}-M_{\mathrm{N}}^{2}} \mathrm{~d} E_{i}=\left(M_{\mathrm{N}}+T_{i}\right) \sqrt{T_{i}\left(2 M_{\mathrm{N}}+T_{i}\right)} \mathrm{d} T_{i}
$$

and get

$$
\Gamma_{\mathrm{N}}=\frac{8}{\pi} \sum_{\substack{j_{\mathrm{N}} t_{1} t_{2} \\ \kappa_{1} \kappa_{2} J}} \frac{\hat{J}^{2}}{\hat{j}_{\Lambda}^{2}} \int \mathrm{d} T_{1} \mathrm{~d} T_{2} \delta\left(\Delta_{j_{\mathrm{N}}}-T_{1}-T_{2}\right) \rho\left(T_{1}, T_{2}\right)\left|\langle\bar{F}|\Delta| I\rangle_{J}\right|^{2},
$$

where

$$
\rho\left(T_{1}, T_{2}\right)=\left(M_{\mathrm{N}}+T_{1}\right) \sqrt{T_{1}\left(2 M_{\mathrm{N}}+T_{1}\right)}\left(M_{\mathrm{N}}+T_{2}\right) \sqrt{T_{2}\left(2 M_{\mathrm{N}}+T_{2}\right)} .
$$

After integrating over $T_{2}$ we are left with the $T_{1}$ integration only,

$$
\Gamma_{\mathrm{N}}=\frac{8}{\pi} \sum_{\substack{j_{\mathrm{N}} t_{1} t_{2} \\ \kappa_{1} \kappa_{2} J}} \frac{\hat{j}^{2}}{\hat{j}_{\Lambda}^{2}} \int_{0}^{\Delta_{j_{\mathrm{N}}}} \mathrm{d} T_{1} \rho\left(T_{1}, T_{2}\right)\left|\langle\bar{F}|\Delta| I\rangle_{J}\right|_{T_{2}=\Delta_{j_{\mathrm{N}}}-T_{1}}^{2} .
$$

As indicated in equation (46), the direct matrix element is given by equation (44) and the exchange term is obtained through the transposition $\left(\kappa_{1}, p_{1}, t_{1}\right) \leftrightarrow\left(\kappa_{2}, p_{2}, t_{2}\right)$.

\subsection{Hypernuclei with open-shell cores and without recoil}

So far everything was done in the strict framework of relativistic physics. In what follows we will make use of analogies with NR calculations. From previous works [19-24] done by our group, we know that to describe the hypernuclei with open-shell cores within the IPSM it is enough to do the following replacement in equation (50)

$$
\frac{\hat{J}^{2}}{\hat{j}_{\Lambda}^{2}} \rightarrow F_{J}^{j_{\mathrm{N}}},
$$

where the spectroscopic factor is given by

$F_{J}^{j_{\mathrm{N}}}=\hat{J}^{-2} \sum_{J_{\mathrm{F}}}\left|\left\langle J_{\mathrm{I}} \|\left(a_{j_{\mathrm{N}}}^{\dagger} a_{j_{\Lambda}}^{\dagger}\right)_{J}|| J_{\mathrm{F}}\right\rangle\right|^{2}=\hat{J}^{2} \sum_{J_{\mathrm{F}}}\left\{\begin{array}{ccc}J_{\mathrm{C}} & J_{\mathrm{I}} & j_{\Lambda} \\ J & j_{\mathrm{N}} & J_{\mathrm{F}}\end{array}\right\}^{2}\left|\left\langle J_{\mathrm{C}}\left\|a_{j_{\mathrm{N}}}^{\dagger}\right\| J_{\mathrm{F}}\right\rangle\right|^{2}$.

As mentioned previously, to evaluate the spectroscopic amplitudes $\left\langle J_{\mathrm{C}}\left\|a_{j_{\mathrm{N}}}^{\dagger}\right\| J_{\mathrm{F}}\right\rangle$, instead of employing the c.f.p. [55] that have been thoroughly used in, both NR [9], and relativistic [4345] calculations, we use the second quantization formalism. In equation (52), the summation goes only over the values of $J_{\mathrm{F}}$ that fulfill the constraint $\left|J_{\mathrm{C}}-j_{\mathrm{N}}\right| \leqslant J_{\mathrm{F}} \leqslant J_{\mathrm{C}}+j_{\mathrm{N}}$. The values 
for $J_{\mathrm{I}}$ and $J_{\mathrm{C}}$ are taken from experimental data and, for most hypernuclei of interest, are listed in table I of [21]. The resulting factors $F_{J}^{j_{N}}$ are listed in table II of the same paper.

Therefore, when the recoil effect is not taken into account, the NMWD transition rate in open shell hypernuclei reads

$$
\Gamma_{\mathrm{N}}=\frac{8}{\pi} \sum_{\substack{j_{\mathrm{N}} t_{1} t_{2} \\ \kappa_{1} \kappa_{2} J}} F_{J}^{j_{\mathrm{N}}} \int_{0}^{\Delta_{j_{\mathrm{N}}}} \mathrm{d} T_{1} \rho\left(T_{1}, T_{2}\right)\left|\langle\bar{F}|\Delta| I\rangle_{J}\right|_{T_{2}=\Delta_{j_{\mathrm{N}}}-T_{1}}^{2} .
$$

We note that, while equation (50) is only valid for doubly closed-shell hypernuclei, equation (53) is valid for both closed- and open-shell hypernuclei.

\subsection{Inclusion of recoil}

As seen above, when recoil is neglected one can perform first the full angular integration $\int \mathrm{d} \hat{\mathbf{p}}_{1} \int \mathrm{d} \hat{\mathbf{p}}_{2}$, leading to a great simplification of the resulting expression. It is self-evident that this cannot be done anymore in the presence of the recoil energy

$$
E_{\mathrm{R}}=\sqrt{M_{\mathrm{R}}^{2}+p_{1}^{2}+p_{2}^{2}+2 p_{1} p_{2} \cos \theta_{12}},
$$

where $M_{\mathrm{R}}$ is the relativistic rest mass of the recoiling nucleus. However, once the hypernucleus is unpolarized (and unaligned), there is no preferred axis along which to orient vectors. Therefore, we can choose to orient $\mathbf{p}_{2}$ with respect to $\mathbf{p}_{1}$ and write

$$
\begin{aligned}
\int \mathrm{d} \hat{\mathbf{p}}_{1} \int \mathrm{d} \hat{\mathbf{p}}_{2} \ldots & =\int \mathrm{d} \hat{\mathbf{p}}_{1} \int \mathrm{d} \hat{\mathbf{p}}_{12} \ldots \\
& =\int \mathrm{d} \phi_{1} \int \mathrm{d} \cos \theta_{1} \int \mathrm{d} \phi_{12} \int \mathrm{d} \cos \theta_{12} \ldots
\end{aligned}
$$

Consequently, we can use equation (23) for integration on $\hat{\mathbf{p}}_{1}$ and equation (24) for integration on $\hat{\mathbf{p}}_{12}$, with the result that, as shown in appendix C, instead of equation (31) we have now $S^{\pi}\left(p_{1} t_{1}, p_{2} t_{2}\right)=\frac{(4 \pi)^{2}}{2} \sum_{\kappa_{1} \kappa_{2} J} \hat{J}^{2} \int \mathrm{d} \cos \theta_{12} \delta\left(\Delta_{j_{\mathrm{N}}}-T_{1}-T_{2}-T_{\mathrm{R}}\right)\left|\left\langle F\left|\Delta^{\pi}\right| I\right\rangle_{J}\right|^{2}$.

From comparison with (31) one concludes that the results developed so far hold valid even when the recoil effect is included, as long as one makes the replacement:

$$
\begin{aligned}
\int p_{1}^{2} \mathrm{~d} p_{1} p_{2}^{2} \mathrm{~d} p_{2} \delta\left(\Delta_{j_{\mathrm{N}}}-T_{1}-T_{2}\right) \ldots \rightarrow & \frac{1}{2} \int \mathrm{d} \operatorname{dos} \theta_{12} p_{1}^{2} \mathrm{~d} p_{1} p_{2}^{2} \mathrm{~d} p_{2} \\
& \times \delta\left(\Delta_{j_{\mathrm{N}}}-T_{1}-T_{2}-T_{\mathrm{R}}\right) \ldots .
\end{aligned}
$$

For the sake of convenience we will work here with the NR limit for the kinetic energy of recoil:

$T_{\mathrm{R}}=E_{\mathrm{R}}-M_{\mathrm{R}} \cong \frac{p_{1}^{2}+p_{2}^{2}+2 p_{1} p_{2} \cos \theta_{12}}{2 M_{\mathrm{R}}} \cong \frac{M_{\mathrm{N}}}{M_{\mathrm{R}}}\left(T_{1}+T_{2}+2 \sqrt{T_{1} T_{2}} \cos \theta_{12}\right)$,

which we consider to be good enough for the present purposes. Moreover, we neglect the binding energy of the recoiling nucleus, and take $M_{\mathrm{R}}=M_{\mathrm{N}}(A-2)$. The transition rate becomes then

$\Gamma_{\mathrm{N}}=\frac{4}{\pi} \sum_{\substack{j_{\mathrm{N}} t_{1} t_{2} \\ \kappa_{1} \kappa_{2} J}} F_{J}^{j_{\mathrm{N}}} \int \mathrm{d} \cos \theta_{12} \mathrm{~d} T_{1} \mathrm{~d} T_{2} \delta\left(\Delta_{j_{\mathrm{N}}}-T_{\mathrm{R}}-T_{1}-T_{2}\right) \rho\left(T_{1}, T_{2}\right)\left|\langle\bar{F}|\Delta| I\rangle_{J}\right|^{2}$. 
To perform the integration on $T_{2}$ we introduce an auxiliary variable $x$, defined as $T_{2}=p_{2}^{2} /\left(2 M_{\mathrm{N}}\right) \equiv x^{2}$, i.e.,

$\delta\left(T_{2}+T_{1}+T_{\mathrm{R}}-\Delta_{j_{\mathrm{N}}}\right) \mathrm{d} T_{2}=\frac{A-2}{A-1} \frac{2 x \mathrm{~d} x}{\left|x^{+}-x^{-}\right|}\left[\delta\left(x-x^{+}\right)+\delta\left(x-x^{-}\right)\right]$,

where

$$
x^{ \pm}=\frac{\sqrt{T_{1}} \cos \theta_{12}}{A-1} \pm \sqrt{\frac{T_{1} \cos ^{2} \theta_{12}}{(A-1)^{2}}+\Delta_{j_{\mathrm{N}}} \frac{A-2}{A-1}-T_{1}}
$$

Therefore

$$
\begin{aligned}
\Gamma_{\mathrm{N}}= & \frac{8}{\pi} \frac{A-2}{A-1} \sum_{\substack{j_{\mathrm{N}} t_{1} t_{2} \\
\kappa_{1} \kappa_{2} J}} F_{J}^{j_{\mathrm{N}}} \int \mathrm{d} \cos \theta_{12} \mathrm{~d} T_{1} \mathrm{~d} x \times \rho\left(T_{1}, T_{2}\right) \\
& \times \frac{\delta\left(x-x^{+}\right)+\delta\left(x-x^{-}\right)}{\left|x^{+}-x^{-}\right|}\left|\langle\bar{F}|\Delta| I\rangle_{J}\right|^{2}
\end{aligned}
$$

After integrating over $x$ one gets

$$
\Gamma_{\mathrm{N}}=\frac{4}{\pi} \sum_{\substack{j_{\mathrm{N}} t_{1} t_{2} \\ \kappa_{1} \kappa_{2} J}} F_{J}^{j_{\mathrm{N}}} \int \mathrm{d} T_{1} \mathrm{~d} \cos \theta_{12}\left\{\left[\rho^{\prime}\left(T_{1}, T_{2}, \cos \theta_{12}, \Delta_{j_{\mathrm{N}}}\right)\left|\langle\bar{F}|\Delta| I\rangle_{J}\right|^{2}\right]_{x \rightarrow x^{+}}+[\cdot]_{x \rightarrow x^{-}}\right\},
$$

where

$$
\rho^{\prime}\left(T_{1}, T_{2}, \cos \theta_{12}, \Delta_{j_{\mathrm{N}}}\right)=\frac{x(A-2) \rho\left(T_{1}, T_{2}\right)}{\sqrt{T_{1} \cos ^{2} \theta_{12}+\Delta_{j_{\mathrm{N}}}(A-2)(A-1)-T_{1}(A-2)^{2}}} .
$$

It is worth mentioning that:

- In the analogous NR formulation [21], it has been shown numerically that the contribution corresponding to the second term in equation (63) is negligibly small compared to that of the first term. This also occurs here.

- In the limit $A \rightarrow \infty$, the result in equation (53) is recovered. Indeed, once

$$
x^{ \pm} \underset{A \rightarrow \infty}{\longrightarrow} \pm \sqrt{\Delta_{j_{\mathrm{N}}}-T_{1}}
$$

$x^{-}$becomes nonphysical. Therefore, the only contribution comes from the first term in equation (63), and, as can be seen from equation (64):

$$
\int \mathrm{d} \cos \theta_{12} \rho^{\prime}\left(T_{1}, T_{2}, \cos \theta_{12}, \Delta_{j_{\mathrm{N}}}\right) \underset{A \rightarrow \infty}{\longrightarrow} 2 \rho\left(T_{1}, T_{2}\right)
$$

\section{Numerical results}

We present here results for the NMWD rates of ${ }_{\Lambda}^{12} \mathrm{C}$. In general, recoil effects can be neglected, as we have learned in previous NR calculations [19, 25], although they are relevant for the energy distribution of emitted particles in very light systems, such as s-shell hypernuclei. They are also crucial for the angular distribution in general, but less important for the 
integrated rates. Therefore equation (53) can be used. However, for reasons of completeness, a few results with the effect of recoil included are also presented.

Two approaches have been tested for the propagators $\Delta^{\mathrm{M}}(|\mathbf{x}-\mathbf{y}|)$, both based on the fact that the ranges of Yukawa-like baryon-baryon forces within hypernuclei depend not only on the intermediate meson mass but also on the baryon masses, as stated in appendix $G$ of [67], namely:

RA1 This is the standard approach in NR calculations $[13,15,68]$, where the energy $q_{0}$ carried by the exchanged meson is constant and always smaller that the meson mass $m_{\mathrm{M}}$, having the value $q^{0}=\Delta / 2=88.5 \mathrm{MeV}$. This implies that the factor $\sqrt{m_{\mathrm{M}}^{2}-\left(q^{0}\right)^{2}}$ in (14) is taking the place of the effective mass $\tilde{m}_{\mathrm{M}}=\sqrt{m_{\mathrm{M}}^{2}-\Delta^{2} / 4}$.

RA2 This is the approach introduced in [44, 45], which is more appropriate for relativistic calculations, where $q_{0}$ is evaluated for each value of the kinetic energy $T_{1}$, with direct and exchange energies being respectively $q_{0}^{D}=\Delta+\varepsilon_{j_{\Lambda}}-T_{1}$, and $q_{0}^{E}=T_{1}-\varepsilon_{j_{\mathrm{N}}}$. Since for the NMWD in $\Lambda$-hypernuclei the energy transfer is of the order $50-150 \mathrm{MeV}, q_{0}$ can be larger than $m_{\pi}$ and the factor $\sqrt{m_{\pi}^{2}-q_{0}^{2}}$ can become complex. Therefore, in the case of the $\pi$ meson, besides making use of equation (14), one also needs equation (15). We are particularly interested in this approach, since, as mentioned above, the energy transfer in the NMWD of charmed nuclei can be large.

Effects due to short-range correlations (SRC) on the initial and final states involved in the NMWD matrix elements in equations (7) and (8) are introduced by making the substitution

$$
\Delta^{\mathrm{M}}(\mathbf{r}) \rightarrow \Delta^{\mathrm{M}}(\mathbf{r}) g_{i}(r) g_{f}(r)
$$

in equation (34), where $r \equiv|\mathbf{x}-\mathbf{y}|$ and

$$
\begin{aligned}
& g_{i}(r)=\left(1-\mathrm{e}^{-r^{2} / \alpha^{2}}\right)^{2}+\beta r^{2} \mathrm{e}^{-r^{2} / \gamma^{2}}, \\
& g_{f}(r)=1-j_{0}\left(q_{\mathrm{c}} r\right)
\end{aligned}
$$

are Jastrow factors corresponding, respectively, to the initial and final states, with $\alpha=0.5 \mathrm{fm}$, $\beta=0.25 \mathrm{fm}^{-2}$, and $\gamma=1.28 \mathrm{fm}$, and $q_{\mathrm{c}}=3.93 \mathrm{fm}^{-1}[13,15,43-45]$. The dipole form-factor cutoffs $\Lambda_{\pi}=1.3 \mathrm{GeV}$ and $\Lambda_{K}=1.2 \mathrm{GeV}$ are also the same as in those works. Jastrow factors have been recently used in relativistic calculations in [69-71] — see also [72] for a discussion on this. Although the use of such factors cannot be a substitute for a relativistic many-body calculation a la Brueckner-Hartree-Fock (RBHF), they are nevertheless useful to assess order of magnitude effects one can expect from SRC. For a very recent discussion on the status of a RBHF theory for finite nuclei, see [73].

We present here two different sorts of comparisons involving our results for the decay rates of ${ }_{\Lambda}^{12} \mathrm{C}$. First, in table 2 , the two relativistic calculations RA1 and RA2 are compared with each other, and also with the analogous NR calculation using the RA1 approach for the propagator. The NR calculation is analogous to the relativistic one in the sense that it uses: the same OME model, the same Jastrow-like correlation function (68), the same single-particle energies, and single-particle wave functions of a harmonic oscillator potential with size parameter $b=1.60 \mathrm{fm}$, which gives the same root-mean-square radius for the initial hypernucleus as the relativistic wave functions. We show the decay rates $\Gamma_{n}$ and $\Gamma_{p}$, the total onenucleon-induced nonmesonic decay rates $\Gamma_{n m}=\Gamma_{n}+\Gamma_{p}$, and the ratios $\Gamma_{n} / \Gamma_{p}$ within different OME models, namely, the $\pi$ and $(\pi, K)$ exchanges without and with SRC. Obviously, the relativistic calculations were evaluated in the laboratory frame of reference (LFR). Therefore, we confront them with NR calculations that also were done in the LFR. These, in turn, have 
Table 2. Comparison between the nonrelativistic (NR) and relativistic results for $\Gamma_{n}, \Gamma_{p}$, $\Gamma_{n m}=\Gamma_{n}+\Gamma_{p}$, and $\Gamma_{n} / \Gamma_{p}$ in ${ }_{\Lambda}^{12} \mathrm{C}$, for different OME models: $\pi$ and $\pi+K$ exchanges without and with SRC. Parity-conserving (PC) and parity-violating (PV) parts of $\Gamma_{n}$ and $\Gamma_{p}$ are given separately. The decay rates are in units of the free $\Lambda$ decay rate, $\Gamma_{\Lambda}^{\text {free }}=2.50 \times 10^{-12} \mathrm{MeV}$. Except for the last row labeled as $(\pi+K)_{\mathrm{r}}$, results were obtained without taking recoil into account.

\begin{tabular}{lcccccc}
\hline Model & $\Gamma_{n}^{(\mathrm{PC})}$ & $\Gamma_{n}^{(\mathrm{PV})}$ & $\Gamma_{p}^{(\mathrm{PC})}$ & $\Gamma_{p}^{(\mathrm{PV})}$ & $\Gamma_{n} / \Gamma_{p}$ & $\Gamma_{n m}$ \\
\hline NR & & & & & & \\
No SRC & & & & & & \\
$\pi$ & 0.11 & 0.16 & 0.87 & 0.40 & 0.21 & 1.54 \\
$\pi+K$ & 0.03 & 0.19 & 0.37 & 0.35 & 0.30 & 0.94 \\
SRC & & & & & & \\
$\pi$ & 0.01 & 0.18 & 0.81 & 0.45 & 0.15 & 1.45 \\
$\pi+K$ & 0.02 & 0.19 & 0.38 & 0.40 & 0.27 & 0.99 \\
\hline RA1 & & & & & & \\
No SRC & & & & & & \\
$\pi$ & 0.12 & 0.15 & 0.69 & 0.33 & 0.26 & 1.29 \\
$\pi+K$ & 0.06 & 0.24 & 0.40 & 0.38 & 0.38 & 1.08 \\
SRC & & & & & & \\
$\pi$ & 0.10 & 0.12 & 0.52 & 0.24 & 0.29 & 0.98 \\
$\pi+K$ & 0.06 & 0.16 & 0.35 & 0.27 & 0.35 & 0.84 \\
\hline RA2 & & & & & & \\
No SRC & & & & & & \\
$\pi$ & 0.17 & 0.22 & 0.79 & 0.45 & 0.31 & 1.63 \\
$\pi+K$ & 0.10 & 0.31 & 0.48 & 0.51 & 0.41 & 1.40 \\
SRC & & & & & & \\
$\pi$ & 0.14 & 0.19 & 0.62 & 0.36 & 0.34 & 1.31 \\
$\pi+K$ & 0.10 & 0.23 & 0.44 & 0.39 & 0.40 & 1.16 \\
$(\pi+K)_{\mathrm{r}}$ & 0.09 & 0.25 & 0.38 & 0.42 & 0.42 & 1.14 \\
\hline & & & & & &
\end{tabular}

been shown elsewhere $[61,62]$ to nicely agree with the NR evaluation in the center-of-mass frame (CMF).

It is not possible here to separate the decay rates $\Gamma_{n}$ and $\Gamma_{p}$ in the usual Block-Dalitz channels [74]:

$$
\begin{array}{lll}
\mathrm{a} \doteq{ }^{1} \mathrm{~S}_{0} \rightarrow{ }^{1} \mathrm{~S}_{0}, & \mathrm{~b} \doteq{ }^{3} \mathrm{P}_{0} \rightarrow{ }^{1} \mathrm{~S}_{0}, & \mathrm{c} \doteq{ }^{3} \mathrm{~S}_{1} \rightarrow{ }^{3} \mathrm{~S}_{1}, \\
\mathrm{~d} \doteq{ }^{3} \mathrm{D}_{1} \rightarrow{ }^{3} \mathrm{~S}_{1}, & \mathrm{e} \doteq{ }^{1} \mathrm{P}_{1} \rightarrow{ }^{3} \mathrm{~S}_{1}, & \mathrm{f} \doteq{ }^{3} \mathrm{P}_{1} \rightarrow{ }^{3} \mathrm{~S}_{1},
\end{array}
$$

as one can always do in the CMF within the s-wave approximation [22]. Therefore, we only show separate results for the PC and PV parts of the decay rates, which contain, respectively, the $(a+c+d)$ and $(b+e+f)$ contributions. From table 2, it can be concluded that:

(i) There is gross agreement between analogous NR and relativistic results, with and without SRC.

(ii) The SRC, while not crucial for some decay rates, can significantly reduce others, both in the NR and in the relativistic cases.

(iii) The decay rates $\Gamma_{n}$ and $\Gamma_{p}$ are both higher in RA2 than in RA1.

(iv) Relativity tends to make $\Gamma_{n}$ become larger and $\Gamma_{p}$ smaller, and this effect is more pronounced in the RA2 approach for the propagator. As a consequence the relativistic 
Table 3. Nonmesonic decay rates $\Gamma_{n}, \Gamma_{p}$ and $\Gamma_{n m}=\Gamma_{n}+\Gamma_{p}$, and the ratio $\Gamma_{n} / \Gamma_{p}$ in ${ }_{\Lambda}^{12} \mathrm{C}$ for several OME models: $\pi$ and $\pi+K$ exchanges without and with SRC. Results obtained with approach RA2 for the propagators are compared with those of the literature using PS coupling and with experimental data. All results are in units of $\Gamma_{\Lambda}^{\text {free }}=2.50 \times 10^{-12} \mathrm{MeV}$.

\begin{tabular}{|c|c|c|c|c|}
\hline Model & $\Gamma_{n}$ & $\Gamma_{p}$ & $\Gamma_{n} / \Gamma_{p}$ & $\Gamma_{n m}$ \\
\hline \multicolumn{5}{|l|}{$\underline{\pi}$} \\
\hline Present (RA2) & 0.39 & 1.24 & 0.31 & 1.63 \\
\hline$[43]$ & 0.27 & 1.32 & 0.20 & 1.62 \\
\hline [45] PS & 0.86 & 2.09 & 0.41 & 2.95 \\
\hline \multicolumn{5}{|l|}{$\pi+\mathrm{SRC}$} \\
\hline$\overline{\text { Present (RA2) }}$ & 0.33 & 0.98 & 0.34 & 1.31 \\
\hline$[43]$ & 0.06 & 0.29 & 0.20 & 0.35 \\
\hline [45] PS & 0.71 & 1.72 & 0.34 & 2.43 \\
\hline \multicolumn{5}{|l|}{$\underline{\pi+K}$} \\
\hline$\overline{\text { Present }}($ RA2) & 0.41 & 0.98 & 0.41 & 1.39 \\
\hline [45] PS & 1.25 & 1.60 & 0.78 & 2.85 \\
\hline \multicolumn{5}{|l|}{$\pi+K+\mathrm{SRC}$} \\
\hline Present (RA2) & 0.33 & 0.82 & 0.40 & 1.15 \\
\hline [43] & 0.05 & 0.36 & 0.15 & 0.41 \\
\hline [45] PS & 0.96 & 1.42 & 0.68 & 2.39 \\
\hline \multicolumn{5}{|l|}{ Experiment } \\
\hline$\overline{[56]}$ & $0.23 \pm 0.08$ & $0.45 \pm 0.10$ & - & - \\
\hline [57] & - & - & $0.51 \pm 0.13 \pm 0.05$ & - \\
\hline [58] & - & - & - & $0.828 \pm 0.056 \pm 0.066$ \\
\hline [59] & - & - & - & $0.953 \pm 0.032$ \\
\hline [60] & - & $0.65 \pm 0.19$ & - & - \\
\hline
\end{tabular}

$n / p$ ratio becomes significantly larger than the NR one, especially when the recoil effect is considered. Therefore, the relativistic approach RA2 helps to solve the longstanding puzzle on the $\Gamma_{n} / \Gamma_{p}$ ratio $[13,75]$. (See also table 3 .)

(v) Inclusion of recoil gives a relatively small contribution, as indicated by the last row. As pointed out elsewhere [23], the effect of recoil is much more relevant in s-shell hypernuclei. Very likely, it is also sizeable in the case of charmed nuclei, and this was the main reason for discussing it in section 2.3.

In table 3 we compare the RA 2 calculations for ${ }_{\Lambda}^{12} \mathrm{C}$ with previous relativistic calculations performed in [43, 45] for several OME models. We consider only the calculations using pseudo-scalar couplings. The difference with the calculation of [45] is due to differences in parametrization and to the fact of a missing normalization factor of $1 / \sqrt{2}$ in the antisymmetrized matrix elements in that work-compare equation (46) here and equation (33) in [45]. This explains why the rates obtained in that reference are approximately twice as large as ours. The results in [43] agree well with ours without SRC, but they disagree when including them; we note that in that reference, separate multipole expansions are made for the propagators and the correlation functions, while here a single multipole expansion is made for the product of these quantities, with coefficients computed numerically as indicated in 
equation (35). We have checked the stability of our results with respect to the number of partial waves and Gauss quadrature points used in the numerical integrations.

In the same table 3 are shown the pertinent experimental data obtained by the KEK and FINUDA groups [56-60], which show good agreement with the present evaluation within the $\pi+K+$ SRC model. In particular, the good agreement for the ratio $\Gamma_{n} / \Gamma_{p}$ should be highlighted. The only significant discrepancy is with the experimental values for $\Gamma_{n}$ and $\Gamma_{p}$ obtained by KEK in [56]. However, there is agreement with the experimental value for $\Gamma_{p}$ obtained by FINUDA group in [60]. As to the last column in tables 2 and 3, it is important to remark that, while all the listed calculations include only one-nucleon-induced transitions, the experimental values include also eventual two-nucleon-induced contributions.

\section{Summary and final remarks}

Starting from the Fermi golden rule for the decay rate, equation (4), we have constructed in section 2 a relativistic formalism to describe the NMWD of single- $\Lambda$ hypernuclei within the framework of the IPSM, with the dynamics represented by the $(\pi, K)$ OME model. First, in section 2.1 we implemented the formalism for hypernuclei whose cores have only closed subshells and neglected recoil effects. Here, the Dirac plane waves are expanded in spherical partial waves, the multipole expansion of the propagator is performed, and the two-body matrix element is properly antisymmetrized with regard to the two outgoing nucleons. Making use of the orthogonality condition given in equation (23) and exploiting the energy conserving $\delta$-function, the six momentum-space integrals in equation (4) are reduced to a single integral in equation (50), with the latter performed numerically. Next, the derived result is generalized to include hypernuclei with open-shell cores. This is done by means of the spectroscopic factors given by equation (52), which are evaluated in second quantization, without recurring to the c.f.p. technique. In this way, we arrive at equation (53). Finally, in section 2.3, we discuss recoil effects, which are important [23] not only for the evaluation of angular distributions of the pairs of emitted nucleons, but also for the study of single kinetic energy spectra in light and medium-weight hypernuclei.

Numerical results for ${ }_{\Lambda}^{12} \mathrm{C}$ are presented in section 3, from where the following conclusions can be drawn. First, table 2 shows the comparison between analogous NR and relativistic calculations of the transition rates $\Gamma_{n}$ and $\Gamma_{p}$. The PC and PV contributions are given separately. Indeed, because of the relatively low energy exchange in the nonmesonic decay, a relativistic formalism by itself is not to bring pronounced effects if implemented correctly. Nevertheless, such a comparison, never done before in the literature, is useful for assessing the ability of a relativistic model to describe experimental data. The agreement between the two formalisms is satisfactory, with the ratio $\Gamma_{n} / \Gamma_{p}$ being appreciably higher in the relativistic calculation and agreeing better with experiment than the NR one, especially when the RA2 approach for the propagators is used; the agreement with experimement is even better when recoil is considered. In addition, the results shown in the table 3 indicate that the $\pi+K+$ SRC OME model provides results that compare well with experimental data.

The present formalism needs to be complemented with a more detailed study of SRC. Proper treatment of SRC is a key issue in nuclear physics, not only in the relativistic context -for recent reviews, see e.g. [76-78]. In the absence of a fully RBHF calculation of finite nuclei [73], a particularly interesting way to treat SRC is provided by the unitary correlation operator method (UCOM) [79]. In the UCOM, a unitary correlation operator moves a pair of nucleons away from each other whenever they start to overlap and, due its unitary character, conserves the probability normalization of the pair relative wave functions. Very recently 
[80], the UCOM has been implemented in a relativistic mean-field model like the one we used in the present paper. As shown in [80], SRC can be handled even in the presence of mesonbaryon form factors within the UCOM and so the method seems to provide a good starting point to include in a consistent manner SRC in relativistic NMWD matrix elements. Work in this direction is in progress, where we also intend to investigate further observables in the NMWD, as the decay asymmetry.

Finalizing, we have achieved our goal of developing a relativistic model that is able to give a reasonable description of NMWD rates of $\Lambda$-hypernuclei, which can be extended for similar weak decays in charmed nuclei, where it is imperative to resort to a relativistic formalism.

\section{Acknowledgments}

Work partially supported by the Argentinean agencies Consejo Nacioal de Investigaciones Científicas y Técnicas-CONICET, Grant No. PIP 0377 (FK), and Fondo para la Investigación Científica y Tecnólogica-FONCYT, Grant No. PICT-2010-2680 (FK), as well as by the Brazilian agencies Fundação de Amparo à Pesquisa do Estado de São Paulo-FAPESP, Grants 2013/01790-5 (FK) and No. 2013/01907-0 (GK), and Conselho Nacional de Desenvolvimento Científico e Tecnológico-CNPq, Grant No.305894/2009-9 (GK). The work of CEF was supported by a post-graduate scholarship from Universidade Estadual Paulista. FK thanks Eulogio Oset for very useful discussions.

\section{Appendix A. Derivation of equation (24)}

From the definition (17) it follows that

$$
\begin{aligned}
2 \int & \sum_{s m} \mathrm{~d} \hat{\mathbf{p}} \hat{j}^{-2} \delta_{j j^{\prime}}\langle\hat{\mathbf{p}} s \mid \kappa m\rangle^{*}\left\langle\hat{\mathbf{p}} s \mid \kappa^{\prime} m\right\rangle \cdots \\
& =2 \hat{j}^{-2} \delta_{j j}(4 \pi)^{2} \int \mathrm{d} \hat{\mathbf{p}} \sum_{s m} i^{l-l^{\prime}} \sum_{\mu}\left(l \mu \frac{1}{2} s \mid j m\right) Y_{l \mu}^{*}(\hat{\mathbf{p}}) \sum_{\mu^{\prime}}\left(l^{\prime} \mu^{\prime} \frac{1}{2} s \mid j m\right) Y_{l^{\prime} \mu^{\prime}}(\hat{\mathbf{p}}) \cdots \\
& =2(4 \pi)^{2} \hat{l}^{-2} \delta_{j j^{\prime}} \delta_{l l^{\prime}} \int \mathrm{d} \hat{\mathbf{p}} \sum_{\mu} Y_{l \mu}^{*}(\hat{\mathbf{p}}) Y_{l \mu}(\hat{\mathbf{p}}) \cdots
\end{aligned}
$$

Now, if we use the relation

$$
4 \pi \sum_{\mu} Y_{l \mu}^{*}(\hat{\mathbf{p}}) Y_{l \mu}(\hat{\mathbf{p}})=\hat{l}^{2},
$$

we can solve the integral over the azimuthal angle to obtain

$$
2 \int \sum_{s m} \mathrm{~d} \hat{\mathbf{p}} \hat{j}^{-2} \delta_{j j}\langle\hat{\mathbf{p}} s \mid \kappa m\rangle^{*}\left\langle\hat{\mathbf{p}} s \mid \kappa^{\prime} m\right\rangle \ldots=\delta_{\kappa \kappa^{\prime}}(4 \pi)^{2} \int_{-1}^{1} \mathrm{~d} \cos \theta \ldots
$$

\section{Appendix B. Relativistic single-particle wave functions}

The evaluation of the matrix elements of the NMWD is made in the context of the IPSM. This means that the $\Lambda$ wave functions are those generated by spherically symmetric mesonic mean fields. That is, in solving the Dirac equations for the single-particle level of $\Lambda$, one must use 
the meson mean fields from the ${ }^{12} \mathrm{C}$ nucleus. This is similar in spirit to the works of Ramos et al [43], where single-particle bound-state wave functions are obtained by solving the Dirac equation with static Lorentz-scalar and -vector Woods-Saxon potentials.

The radial bound-state wave functions $F_{\kappa}(r)$ and $G_{\kappa}(r)$ in (25) and corresponding energy eigenvalues $\varepsilon_{\kappa}$ for a single-particle state $\kappa$ for the $\operatorname{Nor} \Lambda$ are obtained by solving the following Dirac equations:

$$
\begin{aligned}
& \left(\frac{\mathrm{d}}{\mathrm{d} r}+\frac{\kappa}{r}\right) F_{\kappa}+\left(\varepsilon_{\kappa}-V+S\right) G_{\kappa}=0, \\
& \left(\frac{\mathrm{d}}{\mathrm{d} r}-\frac{\kappa}{r}\right) G_{\kappa}-\left(\varepsilon_{\kappa}-V-S\right) F_{\kappa}=0,
\end{aligned}
$$

where the scalar potential $\mathrm{S}=S(r)$ is

$$
S(r)=M+g_{\sigma} \sigma(r),
$$

with $M=M_{\mathrm{N}}$ and $g_{\sigma}=g_{\sigma}^{\mathrm{N}}$ for the $N$, and $M=M_{\Lambda}$ and $g_{\sigma}=g_{\sigma}^{\Lambda}$ for the $\Lambda$; the vector potential $V=V(r)$ for the nucleon is given by

$$
V(r)=g_{\omega}^{\mathrm{N}} \omega_{0}(r)+t_{\kappa} g_{\rho} \rho_{0}(r)+\left(t_{\kappa}+1 / 2\right) e A_{0}(r),
$$

with $t_{\kappa}=1 / 2$ for the proton, $t_{\kappa}=-1 / 2$ for the neutron, and for the $\Lambda$ it is given by

$$
V(r)=g_{\omega}^{\Lambda} \omega_{0}(r) .
$$

The meson and Coulomb fields satisfy the following Klein-Gordon and Poisson equations

$$
\begin{aligned}
\left(-\nabla^{2}+m_{\sigma}^{2}\right) \sigma & =-g_{\sigma}^{\mathrm{N}} \rho_{\mathrm{s}}^{\mathrm{N}}-g_{2} \sigma^{2}-g_{3} \sigma^{3}, \\
\left(-\nabla^{2}+m_{\omega}^{2}\right) \omega_{0} & =g_{\omega}^{\mathrm{N}} \rho_{B}^{\mathrm{N}}, \\
\left(-\nabla^{2}+m_{\rho}^{2}\right) \rho_{0} & =1 / 2 g_{\rho} \rho_{3}, \\
-\nabla^{2} A_{0} & =e \rho_{p},
\end{aligned}
$$

with the densities given by

$$
\begin{aligned}
& \rho_{\mathrm{s}}^{\mathrm{N}}=\sum_{\kappa} \frac{n_{\kappa}^{\mathrm{N}}}{4 \pi r^{2}}\left(\left|F_{\kappa}\right|^{2}-\left|G_{\kappa}\right|^{2}\right), \\
& \rho_{B}^{\mathrm{N}}=\sum_{\kappa} \frac{n_{\kappa}^{\mathrm{N}}}{4 \pi r^{2}}\left(\left|F_{\kappa}\right|^{2}+\left|G_{\kappa}\right|^{2}\right), \\
& \rho_{3}=\sum_{\kappa} \frac{(-)^{t_{\kappa}-1 / 2} n_{\kappa}^{\mathrm{N}}}{4 \pi r^{2}}\left(\left|F_{\kappa}\right|^{2}+\left|G_{\kappa}\right|^{2}\right), \\
& \rho_{p}=\sum_{\kappa} \frac{\left(t_{\kappa}+1 / 2\right) n_{\kappa}^{\mathrm{N}}}{4 \pi r^{2}}\left(\left|F_{\kappa}\right|^{2}+\left|G_{\kappa}\right|^{2}\right),
\end{aligned}
$$

where $n_{\kappa}^{\mathrm{N}}$ are the nucleon occupancies of the state $\kappa$.

The system of equations is solved by iteration following the scheme of [66]:

(i) we solve the Dirac equations for given initial ansätze for the $S$ and $V$ potentials;

(ii) the solutions for $F(r)$ and $G(r)$ are then used to solve the Klein-Gordon and Poisson equations and construct new potentials; and (iii) we put these into the Dirac equations and cycle until convergence to a prescribed precision is attained. Note that the nonlinear terms for the $\sigma$ field are put together with the scalar density $\rho_{\mathrm{s}}^{\mathrm{N}}$ in the iteration procedure. 
Table B1. Single-particle energies for ${ }^{12} \mathrm{C}$ and ${ }_{\Lambda}^{12} \mathrm{C}$. (See text.) Experimental values for ${ }^{12} \mathrm{C}$ are taken from [43], and for ${ }_{\Lambda}^{12} \mathrm{C}$ from [84]. All values are in $\mathrm{MeV}$.

\begin{tabular}{lcc}
\hline Level & Calculated & Experiment \\
\hline$p 1 \mathrm{~s}_{1 / 2}$ & -38.53 & -34. \\
$p 1 \mathrm{p}_{3 / 2}$ & -13.52 & -15.96 \\
$n 1 \mathrm{~s}_{1 / 2}$ & -42.03 & -37. \\
$n 1 \mathrm{p}_{3 / 2}$ & -16.65 & -18.72 \\
$\Lambda 1 \mathrm{~s}_{1 / 2}$ & -11.59 & -10.79 \\
\hline
\end{tabular}

The numerical values of the meson-nucleon parameters are those of the column NL3 [81] of table I in [82], and for the meson-lambda couplings are those from [83] (masses are given in $\mathrm{MeV})$ :

$$
\begin{aligned}
g_{\sigma}^{\mathrm{N}} & =10.2169, g_{\omega}^{\mathrm{N}}=12.8675, \quad g_{\rho}=8.9488, \\
e^{2} / 4 \pi & =1 / 137, g_{\sigma}^{\Lambda}=0.464 g_{\sigma}^{\mathrm{N}}, g_{\omega}^{\Lambda}=0.481 g_{\omega}^{\mathrm{N}}, \\
g_{2} & =-10.4307 \mathrm{fm}^{-1}, \quad g_{3}=-28.8851, \\
m_{\sigma} & =508.1941, \quad m_{\omega}=782.501, \quad m_{\rho}=763.000, \\
M_{\mathrm{N}} & =939, \quad M_{\Lambda}=1116.06 .
\end{aligned}
$$

In table B1, we present the single-particle energies for ${ }^{12} \mathrm{C}$ and ${ }_{\Lambda}^{12} \mathrm{C}$. Note that these results are obtained without adjusting any parameters to fit experimental numbers. Clearly, a reasonable description of the experimental single-particle energies is achieved. Of course, a better description could be obtained by fine tuning the parameters, but for the purposes of the present paper such a refinement is not necessary.

\section{Appendix C. Derivation of equation (56)}

Here we demonstrate the result in equation (56) starting from the definition in equation (28) for $S^{\pi}\left(p_{1} t_{1}, p_{2} t_{2}\right)$, i.e.,

$$
S^{\pi}\left(p_{1} t_{1}, p_{2} t_{2}\right) \equiv \sum_{\substack{m_{\Lambda} m_{\mathrm{N}} \\ s_{1} s_{2}}} \int \mathrm{d} \hat{\mathbf{p}}_{1} \mathrm{~d} \hat{\mathbf{p}}_{2} \delta\left(\Delta_{j_{\mathrm{N}}}-T_{1}-T_{2}-T_{\mathrm{R}}\right)\left|\mathcal{M}_{\mathrm{FI}}^{\pi}\right|^{2} .
$$

Using the expansion in equation (26) and making the change of variable $\hat{\mathbf{p}}_{2} \rightarrow \hat{\mathbf{p}}_{12}$ as shown in equation (55), we are free to perform the $\hat{\mathbf{p}}_{1}$ integration according to equation (23), and are left with

$$
\begin{aligned}
S^{\pi}\left(p_{1} t_{1}, p_{2} t_{2}\right)= & (4 \pi)^{2} \sum_{\substack{m_{\Lambda} m_{\mathrm{N}} \\
\kappa_{1} m_{1} s_{2}}} \int \mathrm{d} \hat{\mathbf{p}}_{12} \delta\left(\Delta_{j_{\mathrm{N}}}-T_{1}-T_{2}-T_{\mathrm{R}}\right) \\
& \times\left|\sum_{\kappa_{2} m_{2}}\left\langle\hat{\mathbf{p}}_{12} s_{2} \mid \kappa_{2} m_{2}\right\rangle\left\langle F\left|\Delta^{\pi}\right| I\right\rangle\right|^{2}
\end{aligned}
$$


Next, we perform the angular momentum algebra as in equation (30),

$$
\begin{aligned}
S^{\pi}\left(p_{1} t_{1}, p_{2} t_{2}\right)= & (4 \pi)^{2} \sum_{\substack{m_{\Lambda} m_{\mathrm{N}} \\
\kappa_{1} m_{1} s_{2}}} \int \mathrm{d} \hat{\mathbf{p}}_{12} \delta\left(\Delta_{j_{\mathrm{N}}}-T_{1}-T_{2}-T_{\mathrm{R}}\right) \\
& \times \sum_{\substack{\kappa_{2} m_{2} \\
J M}}\left\langle\hat{\mathbf{p}}_{12} s_{2} \mid\right\rangle \kappa_{2} m_{2}^{*}\left\langle F\left|\Delta^{\pi}\right| I\right\rangle_{J}^{*}\left(j_{1} m_{1} j_{2} m_{2} \mid J M\right) \\
& \times\left(j_{\Lambda} m_{\Lambda} j_{\mathrm{N}} m_{\mathrm{N}} \mid J M\right) \sum_{\kappa_{2}^{\prime} m_{2}^{\prime}}\left\langle\hat{\mathbf{p}}_{12} s_{2} \mid \kappa_{2}^{\prime} m_{2}^{\prime}\right\rangle\left\langle F^{\prime}\left|\Delta^{\pi}\right| I\right\rangle_{J^{\prime}} \\
& \times\left(j_{1} m_{1} j_{2}^{\prime} m_{2}^{\prime} \mid J^{\prime} M^{\prime}\right)\left(j_{\Lambda} m_{N} j_{\mathrm{N}} m_{\mathrm{N}} \mid J^{\prime} M^{\prime}\right),
\end{aligned}
$$

to obtain

$$
\begin{aligned}
S^{\pi}\left(p_{1} t_{1}, p_{2} t_{2}\right)= & (4 \pi)^{2} \sum_{s_{2}} \int \mathrm{d} \hat{\mathbf{p}}_{12} \delta\left(\Delta_{j_{\mathrm{N}}}-T_{1}-T_{2}-T_{\mathrm{R}}\right) \\
& \times \sum_{\substack{\kappa_{2} m_{2} \\
\kappa_{2}^{\prime} m_{2}^{\prime}}}\left\langle\hat{\mathbf{p}}_{12} s_{2} \mid \kappa_{2} m_{2}\right\rangle^{*}\left\langle\hat{\mathbf{p}}_{12} s_{2} \mid \kappa_{2}^{\prime} m_{2}^{\prime}\right\rangle \sum_{\substack{\kappa_{1} J \\
m_{1} M}}\left(j_{1} m_{1} j_{2} m_{2} \mid J M\right) \\
& \times\left(j_{1} m_{1} j_{2}^{\prime} m_{2}^{\prime} \mid J M\right)\left\langle F\left|\Delta^{\pi}\right| I\right\rangle_{J}^{*}\left\langle F^{\prime}\left|\Delta^{\pi}\right|_{\mathrm{I}}\right\rangle_{J} .
\end{aligned}
$$

Due to the property

$$
\sum_{m_{1} M}\left(j_{1} m_{1} j_{2} m_{2} \mid J M\right)\left(j_{1} m_{1} j_{2}^{\prime} m_{2}^{\prime} \mid J M\right)=\frac{\hat{J}^{2}}{\hat{j}_{2}^{2}} \delta_{m_{2} m_{2}^{\prime}} \delta_{j_{2} j_{2}^{\prime}},
$$

equation (C.4) reduces to

$$
\begin{aligned}
S^{\pi}\left(p_{1} t_{1}, p_{2} t_{2}\right)= & (4 \pi)^{2} \sum_{\kappa_{2} m_{2}} \frac{\delta_{j_{2} j_{2}^{\prime}}}{\hat{j}_{2}^{2}} \int \mathrm{d} \hat{\mathbf{p}}_{12} \delta\left(\Delta_{j_{\mathrm{N}}}-T_{1}-T_{2}-T_{\mathrm{R}}\right) \\
& \times\left\langle\hat{\mathbf{p}}_{12} s_{2} \mid \kappa_{2} m_{2}\right\rangle^{*}\left\langle\hat{\mathbf{p}}_{12} s_{2} \mid \kappa_{2}^{\prime} m_{2}\right\rangle \sum_{\kappa_{1} J} \hat{J}^{2}\left\langle F\left|\Delta^{\pi}\right| I\right\rangle_{J}^{*} \\
& \times\left\langle F^{\prime}\left|\Delta^{\pi}\right| I\right\rangle_{J} .
\end{aligned}
$$

Finally, using equation (24) one gets equation (56).

\section{References}

[1] Botta E, Bressani T and Garbarino G 2012 Eur. Phys. J. A 4841

[2] Feliciello A 2014 Few Body Syst. 55605

[3] Garbarino G 2013 Nucl. Phys. A 914170

[4] Bufalino S 2013 Nucl. Phys. A 914160

[5] Olive K A et al 2014 Chin. Phys. C 38090001

[6] McKellar B H J and Gibson B F 1984 Phys. Rev. C 30322

[7] Dubach J F, Feldman G B, Holstein B R and de la Torre L 1996 Ann. Phys. NY 249146

[8] Halderson D 1993 Phys. Rev. C 48581

[9] Parreño A, Ramos A and Bennhold C 1997 Phys. Rev. C 56339

[10] Itonaga K, Ueda Y and Motoba T 2002 Phys. Rev. C 65034617

[11] Itonaga K, Motoba T and Ueda T Mod. Phys. Lett. A 18135

[12] Itonaga K, Motoba T, Ueda T and Rijken Th A 2008 Phys. Rev. C 77044605

[13] Barbero C et al 2002 Phys. Rev. C 66055209

[14] Krmpotić F and Tadić D 2003 Braz. J. Phys. 33187

[15] Barbero C, De Conti C, Galeão A P and Krmpotić F 2003 Nucl. Phys. A 726267 
[16] Robertson N J and Dickhoff W H 2005 Phys. Rev. C 72024320

[17] Barbero C, Galeão A P and Krmpotić F 2005 Phys. Rev. C 72035210

[18] Barbero C, Galeão A P and Krmpotić F 2007 Phys. Rev. C 76054321

[19] Barbero C, Galeão A P, Hussein M S and Krmpotić F 2008 Phys. Rev. C 78044312

[20] Bauer E, Galeão A P, Hussein M S and Krmpotić F 2010 Nucl. Phys. A 834 599c

[21] Krmpotić F, Galeão A P and Hussein M S 2010 AIP Conf. Proc 124551

[22] Krmpotić F 2010 Phys. Rev. C 82055204

[23] Krmpotić F 2014 Few Body Syst. 55219

[24] Krmpotić F and De Conti C 2014 Int. J. Mod. Phys. E 231450089

[25] Gonzalez I et al 2011 J. Phys. G: Nucl. Part. Phys. 38115105

[26] Tyapkin A A 1975 Yad. Fiz. 22181

[27] Iwao S 1977 Lett. Nuovo Cimento 19647

[28] Dover C B and Kahana S H 1977 Phys. Rev. Lett. 391506

[29] Gatto R and Paccanoni F 1978 Nuovo Cimento A 46313

[30] Kolesnikov N N et al 1981 Sov. J. Nucl. Phys. 34533

[31] Bhamathi G 1981 Phys. Rev. C 241816

[32] Bando H and Bando M 1982 Phys. Lett. B 109164

[33] Gibson B F, Dover C B, Bhamathi G and Lehman D R 1983 Phys. Rev. C 272085

[34] Starkov N I and Tsarev V A 1986 Nucl. Phys. A 450507

[35] Cai C H, Li L, Tan Y H and Ning P Z 2003 Europhys. Lett. 64448

[36] Tsushima K and Khanna F C 2003 Phys. Lett. B 552138

[37] Tsushima K and Khanna F C 2003 Phys. Rev. C 67015211

[38] Tsushima K and Khanna F C 2004 J. Phys. G: Nucl. Part Phys. 301765

[39] Bunyatov S A et al 1992 Sov. J. Part. Nucl. 23253

[40] Batusov Yu et al 1981 JETP Lett 3356

[41] Lyukov V V 1989 Nuovo Cimento A 102583

[42] Brockmann R and Weise W 1977 Phys. Lett. B 69167

[43] Ramos A et al 1991 Phys. Lett. B 264233

Ramos A et al 1992 Nucl. Phys. A 544703

[44] Conti F 2009 A relativistic model for the non-mesonic weak decay of the ${ }^{12} \mathrm{C}$ hypernucleus $P h D$ Thesis University of Pavia, Italy

[45] Conti F, Meucci A, Giusti G and Pacati F D 2009 (arXiv:0912.3630)

[46] Guichon P A M 1989 Phys. Lett. B 200235

Guichon P A M, Saito K, Rodionov E N and Thomas A W 1996 Nucl. Phys. A 601349

[47] Bracco M E, Krein G and Nielsen M 1998 Phys. Lett. B 432258

[48] Tsushima K, Saito K and Thomas A W 1997 Phys. Lett. B 4117

[49] Tsushima K, Saito K, Haidenbauer J and Thomas A W 1998 Nucl. Phys. A 630691

[50] Tsushima K, Saito K and Thomas A W 1997 Phys. Lett. B 4119

[51] Guichon P A M, Thomas A W and Tsushima K 2008 Nucl. Phys. A 81466

[52] Ring P 2000 private communication

[53] Hagino K and Yao J M 2015 Relativistic Density Functional for Nuclear Structure (Singapore: World Scientific)

[54] Almar R, Civitarese O, Krmpotić F and Navaza J 1972 Phys. Rev. C 6187

Navaza J 1972 Descripción de Núcleos Vibracionales con el Modelo Unificado mediante Técnicas Diagramáticas University of La Plata, Argentina

[55] de-Shalit A and Talmi I 1963 Nuclear Shell Theory (New York: Academic)

[56] Kim M et al 2009 Phys. Rev. Lett. 103182502

[57] Kim M J et al 2006 Phys. Lett. B 64128

[58] Sato Y et al 2005 Phys. Rev. C 71025203

[59] Okada S et al 2005 Nucl. Phys. A $754178 \mathrm{c}$

[60] Agnello M et al 2014 Phys. Lett. B 738499

[61] Galeão A P, Barbero C, De Conti C and Krmpotić F 2013 AIP Conf. Proc 1529247

[62] De Conti C, Barbero C, Galeão A P and Krmpotić F 2014 AIP Conf. Proc 1625181

[63] Serot B D and Walecka J D 1986 Adv. Nucl. Phys. 161

[64] Maessen P M M, Rijken Th A and de Swart J J 1989 Phys. Rev. C 402226

[65] Doi M, Kotani T and Takasugi E 1985 Prog. Theor. Phys. Suppl. 831

[66] Horowitz C J and Serot B D 1981 Nucl. Phys. A 368503

[67] Barbero C, Horvat D, Krmpotić F, Narančić Z and Tadić D 2001 Fizika B 101 
[68] Sasaki K, Izaki M and Oka M 2005 Phys. Rev. C 71035502

[69] Panda P K, Menezes D P, Providência C and da Providência J 2005 Phys. Rev. C 71015801

[70] Panda P K, da Providência J and Providência C 2006 Phys. Rev. C 73035805

[71] Panda P K, Providência C and da Providência J 2007 Phys. Rev. C 75065806

[72] Parreño A, Ramos A and Oset E 1997 Phys. Rev. C 512477

[73] Ring P 2015 J. Phys. Conf. Ser 580012005

[74] Block M M and Dalitz R H 1963 Phys. Rev. Lett. 1196

[75] Garbarino G et al 2003 Phys. Rev. Lett. 91112501

Garbarino G et al 2004 Phys. Rev. C 69054603

[76] Frankfurt L, Sargsian M and Strikman M 2008 Int. J. Mod. Phys. A 232991

[77] Arrington J et al 2012 Prog. Part. Nucl. Phys. 67898

[78] Ciofi degli Atti C 2015 Phys. Rep. 5901

[79] Feldmeier H, Neff T, Roth R and Schnack J 1998 Nucl. Phys. A 63261

[80] Hu J, Toki H, Wen W and Shen H 2010 Eur. Phys. J A 43323

[81] Lalazissis G A, König J and Ring P 1997 Phys. Rev. C 55540

[82] Long W, Meng J, Van Giai N and Zhou S-G 2004 Phys. Rev. C 69034319

[83] Rufa M et al 1990 Phys. Rev. C 422469

[84] Chrien R E et al 1979 Phys. Lett. B 8931 